\title{
Residual urinary Incontinence after Successful Repair of Obstetric Fistula
}

\author{
FAHMIDAZABIN ${ }^{1}$, SAYEBAAKHTER ${ }^{2}$, MUSARRAT SULTANA ${ }^{3}$
}

\begin{abstract}
Objective(s): Aim of the study was to identify the risk factors in those women who remain with incontinence after successful fistula repair.

Materials \& method: The women admitted in Dhaka Medical College Hospital with obstetrical genitourinary fistula were the study population.A observational study was done with all women having successful repair. They were asked to return for a follow-up appointment, 3 months after discharge. Women were examined and questioned at discharge and at follow up appointment. A structured questionnaire were used and information entered into a database.

Results: Women who returned for follow-up 3 month postsurgery were included in predictors of closure analyses. Small bladder size (ARR 3.7; 95\% CI 1.2-11.8), severe scarring (ARR 1.2; 95\% Cl 1.1-2.7), urethral involvement (ARR 7.3; 95\% Cl 3.3-1.46), were predicted failed fistula closure. Women with a closed fistula at 3 month follow-up were included in predictors of residual incontinence analyses.

Conclusion:The prognosis for genital fistula closure is related to preoperative bladder size, previous repair, vaginal scarring, and urethral involvement.
\end{abstract}

Key words: Genital fistula, residual urinary incontinence.

\section{Introduction:}

Obstetric fistula surgery presents many challenges. The injury usually occurs in resource-poor areas and is thus managed in hospitals that are themselves poorly resourced. The pathology is complex and extensive, affecting any and sometimes all parts of the lower urinary tract. The long obstructed labour and resulting ischemia throughout the pelvis can destroy all the normal mechanisms for urinary continence. ${ }^{1}$

It is one thing to be able to close the defect and try to restore normal anatomy, but it is quite another thing altogether to obtain a functional closure, ensuring normal continence. There are varied reports about the extent of incontinence after fistula surgery, from $8 \%{ }^{1}$ to more widely accepted figures of $18-$ $33 \%,{ }^{2}$ even up to $47 \%$ in an unpublished survey performed in the Addis Ababa Fistula Hospital in 2003. As with all things, it depends on how closely a fistula surgeon look for the problem. In the early work by Kelly and Kwast, ${ }^{1}$ the figure was the number of women returning to the hospital with continuing incontinence despite a closed fistula. It is likely that many women would have remained at home with mild incontinence. The figures of 18$33 \%$ had accepted way of looking for incontinence including a basic set of structured questions ranging from'are you wet with cough or heavy activity? 'are you leaking urine involuntarily when lying? ${ }^{2}$ A cough examination with a full bladder was used to confirm the diagnosis.

The exact nature of the incontinence is often complex and only a handful of studies have investigated the nature of the pathology. One study ${ }^{3}$ of 22 women with severe incontinence following fistula closure underwent urodynamic assessment: $41 \%$ had genuine stress incontinence (GSI); $14 \%$ had GSI and poor

1. Associate Professor, Dept of OB/GYN, Bangabandhu Sheikh Mujib Medical University

2. Chief Consultant, MAMM'S Institute of Fistula and Women's health

3. Consultant, Bangladesh Police Hospital 
compliance; $41 \%$ had GSI and detrusor overactivity, and $4 \%$ had voiding disorder and overflow incontinence.

The prognostic factors associated with incontinence after successful closure of fistula have been shown in observational studies and include: a) If the urethra is involved in the defect; the strongest determining risk factor with an odds ratio of 8.4. The urethra is involved in some way in up to $63 \%$ of cases (unpublished series, Browning, Barhirdar, Ethiopia). b) If there is significant vaginal scarring, such that a small Sims speculum cannot be inserted into the vagina without relaxing incisions. c) If there is a reduced bladder volume, having capacity less than $100 \mathrm{ml}$.

Purpose of this study was to explore the risk factors in those women who remain with incontinence after fistula surgery in a series of obstetrics fistula.

\section{Methods:}

This prospective observational study was conducted in Obstetric and Gynaecology department of Dhaka Medical College and Hospital (DMCH) between January 2003 and July 2012. There is a unique dedicated fistula repair center in $\mathrm{DMCH}$ where different complicated fistula cases are referred from different parts of the country. Expert senior surgeons are usually do these difficult operations. Four hundred and seventy five (475) women were operated during this period. The patients who had their fistula successfully closed were entered into the study and divided into two groups, those with residual urethral incontinence and those who were fully continent and these cases were followed three months post surgery. The following parameters were recorded for all patients; a) urethral involvement (the urethra was said to be involved if the distance between the external urinary meatus and distal margin of the fistula was $<3.5 \mathrm{~cm}$ ); b) whether surgery was a repeat operation (i.e. either having a previous successful operation and sustaining another obstetric ûstula after a subsequent delivery, c) if the woman had a previous failed repair); d) if the fistula was large $(\geq 6 \mathrm{~cm}) ; e)$ if the bladder was described as being small at the time of operation (if during the intraoperative dye test performed after closure of the fistula, the bladder could not hold the $50 \mathrm{ml}$ instillation of dye); f) if the ureter was outside of the bladder at the time of the operation and needed reimplantation (vaginally); $g$ ) if there was significant vaginal scarring (reflected by the need for a vaginoplasty or large episiotomy to enable the introduction of an Auvards speculum), h) if vaginal reconstruction with either a labial or gluteal flap was needed; i) if a rectovaginal fistula was present; and j) if there were multiple vesicovaginal fistulae present. The woman's age, parity, length of time in labour, time since delivery, diameter of the fistula, delivery method and outcome of the delivery (live birth or stillbirth) were also recorded. Among the 475 patients, 53 were omitted from the study because of incomplete papers(eleven), the operation resulted in breakdown of repair (eighteen) and rest of the fistula was the rectovaginal; fistulas(twenty four). So 422 patients' records were entered into the analysis. Sucessful closure was done in 373 cases. One hundred and thirty one (31\%) women from the sample suffered residual incontinence after the repair.

The results were analyzed using the chi-square test for comparisons of all proportions, the $t$ test for diameter of the fistula, time in labour and age, or the Mann-Whitney $U$ test for all non-normally distributed data (parity and time since delivery). A multivariate analysis was performed on these markers to determine those factors that were significant independent predictors of developing incontinence following fistula repair. AP value $<0.5$ was considered as significant.

\section{Results:}

The occurence of the pre-specified parameters in the two groups of women is shown in Table-I. Statistically significant associations with residual incontinence were seen if the injury had affected the urethra: if it were a repeat fistula repair; if the fistula was large; if the bladder was described as small; if severe scarring required a vaginoplasty or flap vaginal reconstruction. All of these can be regarded as indirect markers of more extensive injuries. A number of patient characteristics, fistula characteristics and context of repair variables were significantly $(p<0.05)$ associated with fistula closure at the 3 month follow-up visit. It was found that small bladder size, prior repair, severe 
Table-I

Measured parameters comparing women with residual incontinence after operative repair and those without

\begin{tabular}{|c|c|c|c|}
\hline & 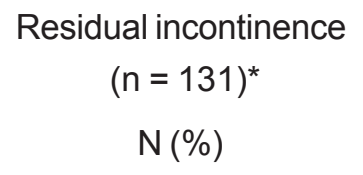 & $\begin{array}{l}\text { No incontinence } \\
\qquad \begin{array}{c}(n=242)^{*} \\
N(\%)\end{array}\end{array}$ & p \\
\hline Urethral involvement & $119(89)$ & $157(64.88)$ & 0.001 \\
\hline Women having repeat procedure ${ }^{* *}$ & $27(20.61)$ & $31(12.80)$ & 0.005 \\
\hline Number of repeats & $1-5$ & $1-3$ & \\
\hline Small bladder & $16(12.21)$ & $4(6.65)$ & 0.001 \\
\hline Ureter outside bladder & $5(3.81)$ & $11(5.55)$ & 0.81 \\
\hline Vaginoplasty at operation & $27(20.61)$ & $77(31.82)$ & 0.001 \\
\hline Rectovaginal fistula present & $17(12.98)$ & $18(7.43)$ & 0.001 \\
\hline \multirow[t]{2}{*}{ Multiple vesicovaginal fistulae } & $9(6.9)$ & $18(7.43)$ & 1.0 \\
\hline & Mean (range) & Mean (range) & \\
\hline Parity & $1.74(1-7)$ & $2.93(1-13)$ & $0.001 \dagger$ \\
\hline Average fistula diameter $(\mathrm{cm})$ & $3.75(1-9)$ & $3.3(0.5-8)$ & $0.001 \#$ \\
\hline Time in labour (days) & $3.05(1-6)$ & $3.16(0.5-8$ & $0.066 \#$ \\
\hline Age (years) & $26.5(19-63)$ & $25.9(14-50)$ & $0.001 \#$ \\
\hline Time since delivery (months) & $26.7(2-563)$ & $18.3(1.5-342)$ & $0.89 \dagger$ \\
\hline Delivery method & $\mathrm{N} \%$ & $\mathrm{~N} \%$ & \\
\hline Vaginal delivery & $117(89.31)$ & 205(84.71) & \\
\hline Caesarean & $10(7.63)$ & $25(10.33)$ & 0.010 \\
\hline Caesarean hysterectomy & $4(3.05)$ & $12(4.96)$ & \\
\hline
\end{tabular}

Statistical tests by chi-square for all except $t$ test \# and Mann-Whitney $U$ test $\dagger$.

Table-II

Significant independent predictors of developing urinary incontinence following fistula repair

\begin{tabular}{lc}
\hline Parameter & Adjusted odds ratio $(95 \% \mathrm{Cl})$ \\
\hline Urethral involvement & $7.3(3.2-14.6)$ \\
Small bladder & $3.7(1.2-11.8)$ \\
Vaginoplasty (vaginal scarring) & $1.2(1.1-2.7)$ \\
Diameter of fistula* & $1.23(1.06-1.33)$ \\
\hline
\end{tabular}

${ }^{*}$ Odds ratio for each centimetre increase in diameter. 
Table-III

Patient and Fistula Characteristics by Fistula Closure and Residual Incontinence at 3 Months Postrepair

\begin{tabular}{|c|c|c|c|}
\hline \multirow[b]{2}{*}{ Characteristics } & \multirow{2}{*}{$\begin{array}{l}\text { Fistula Closure }(\mathrm{n}=422) \\
\text { Closed }\end{array}$} & \multicolumn{2}{|c|}{ Residual Incontinence $(n=131)$} \\
\hline & & $\begin{array}{c}\text { Noresidual } \\
\text { incontinence }\end{array}$ & $\begin{array}{c}\text { Residual } \\
\text { incontinence }\end{array}$ \\
\hline Total & 373 & 242 & 131 \\
\hline \multicolumn{4}{|l|}{ Patient characteristics } \\
\hline Age (mean, SD) & $\begin{array}{c}27.8 \pm 11 \\
N(\%)\end{array}$ & $\begin{array}{l}27.4 \pm 10.8 \\
\quad \mathrm{~N}(\%)\end{array}$ & $\begin{array}{l}29.0 \pm 11.7 \\
N(\%)\end{array}$ \\
\hline Completed primary school or higher & $85(22.8)$ & $38(15.7)$ & $47(35.9)$ \\
\hline Rural residence & $340(91.15)$ & $220(90.90)$ & $120(91.60)^{\dagger}$ \\
\hline Development of fistula in $1^{\text {st }}$ pregnancy & $220(58.98)$ & $141(58.3)$ & $79(60.3)^{\dagger}$ \\
\hline Prior repair & $75(20.1)$ & $27(11.2)$ & $48(36.6)$ \\
\hline \multicolumn{4}{|l|}{ Fistula characteristics } \\
\hline \multicolumn{4}{|l|}{ Location and type } \\
\hline Midvaginal & $114(30.6)$ & $71(29.3)$ & $43(32.82)^{\dagger}$ \\
\hline Juxtaurethral & $83(22.3)$ & $22(9.1)$ & $61(46.6)^{\dagger}$ \\
\hline Juxtacervical & $68(18.2)$ & $33(13.64)$ & $35(26.7)$ \\
\hline Circumferential & $105(28.15)$ & $50(20.66)$ & $55(41.98)^{\dagger}$ \\
\hline Intracervical & $23(6.2)$ & $19(7.9)$ & $4(3.1)^{\dagger}$ \\
\hline \multicolumn{4}{|l|}{ Fistula size $(\mathrm{cm})$} \\
\hline Small (smaller than 2) & $116(31.1)$ & $83(34.3)$ & $33(25.2)$ \\
\hline Medium (2-3) & $179(48)$ & $121(50)$ & $58(44.3)$ \\
\hline Large (4-5) & $64(17.2)$ & $31(12.81)$ & $33(25.2)^{\dagger}$ \\
\hline Extensive (longer than 6) & $22(6)$ & $15(6.2)$ & $7(5.3)^{*}$ \\
\hline \multicolumn{4}{|l|}{ Scarring } \\
\hline No or mild scarring (reference category) & $249(66.9)$ & $171(70.66)$ & $78(59.5)$ \\
\hline Moderate scarring & $104(27.8)$ & $66(27.3)$ & $38(29)$ \\
\hline Severe scarring & $20(5.3)$ & $09(3.8)$ & $14(10.9)^{\dagger}$ \\
\hline
\end{tabular}

vaginal scarring and urethral involvement were found to predict failure of fistula closure at three months post repair.

\section{Discussion}

Urinary incontinence following fistula repair, in particular obstetric fistula continues to be a significant problem $^{1,2}$. A number of papers have described surgical interventions, but often with much less success than that enjoyed in women without fistulas. ${ }^{4-7}$ We found, as others have ${ }^{8,9}$ no relationship between fistula size and successful closure after repair surgery. It is plausible that fistula size, independent of other factors, is not a predictor of repair outcome because even large defects can be surgically closed, whereas other factors such as prior repair or severe scarring (that may reduce the amount of viable tissue), or urethral involvement (that may affect sphincter mechanisms), cannot be easily addressed surgically. Our results also contribute to the growing body of evidence showing that patient characteristics and comorbidities do not independently predict fistula closure or residual incontinence after successful closure. .,9- $^{7}$ 12 Our results provide further evidence supporting the role of vaginal scarring and urethral involvement in predicting failure to close the fistula and residual incontinence following fistula closure. $5,7,8,10,12,13$ They also support the smaller number of studies suggesting a negative prognostic role of bladder size and prior repair ${ }^{6,7,12,13}$ So risk factors identified were 
involvement of the urethra, a small functional bladder capacity, increasing diameter of the fistula and the need for vaginal reconstruction. It is interesting to note that delivery method seemed protective for developing residual incontinence after fistula repair. Having a caesarean section or caesarean hysterectomy seems protective despite the fact that these women also had a long obstructed labour before the operation was performed (average 2.7 days). The lower the obstruction in the pelvis, perhaps the lower the injury to the urinary tract so the urethra is involved and vice versa; for higher obstructions, the higher the injury, thus the urethra is not involved. Indeed, $32 \%$ of the women who had a caesarean section had the urethra involved in the fistula compared with $66 \%$ of women overall, and in the multivariate analysis, the delivery method was not an independent predictor. It was also interesting to note that there was no signiûcant association for a woman to develop residual urinary incontinence with the time elapsed since delivery. It could be expected that after a long wait before treatment, the bladder loses the ability to function in a physiological manner, resulting in incontinence. This does not appear to be the case.

Our data confirms the association of urethral involvement as a risk factor, as previously surmised. The ischemic process during the obstructed labour will affect the continence mechanisms, usually completely destroying them. After the repair of the fisstula and urethra, what remains is a scarred, 'drainpipe' type urethra with no physiological function. It is important to note that circumferential injuries to the urethra were not examined separately in this series. These injuries are more extensive, the urethra or urethral remnant being completely detached from the bladder with extensive tissue loss and scarring in between. If considered separately, this type of injury may be an even stronger predictor of residual incontinence. If the bladder is small after repair, with most of the bladder tissue having been destroyed in the ischemic process, there is little reservoir to be able to store urine and incontinence results. The resulting small bladder is often stiff and noncompliant, presumably due to an increased scar tissue in the bladder wall. Simple cystometry employing a Foley catheter and sterile water instilled into the bladder of these women with a syringe usually reveals a high pressure with only $20-50 \mathrm{ml}$ instilled into the bladder. Vaginal scarring, which is often so severe as to have completely obliterated the vagina, can often cause the urethra to be held open, thus removing any physiological function. Larger fistulae require more extensive dissection to be able to close the defect. This can cause postoperative scarring around the urethra which will cause incontinence in the manner described for vaginal scarring, holding the urethra open. During the repair, there may be some slight tension on the urethra from the suture line, again holding the urethra open. To assist these patients to be completely cured, further attention needs to be given to the repair of fistulae when these identified risk factors are present. These techniques need to be cheap, easy and adaptable to the environments in the developing world where these injuries occur. One such technique is to developed a sling of pubococcygeal muscle beneath the urethra in the women with the above characteristics. ${ }^{2}$ From a personal series of urethral fistulae, the use of this sling had reduced the incidence of urethral incontinence following repair from $55 \%$ (n $=44)$ to $39 \%(n=272)$. Techniques to maintain urethral length for women with urethral injuries also require further research. Simple approximation of the fistula edges to close the defect when the urethra has been partially destroyed may leave the urethral length at a mere $1 \mathrm{~cm}$ and without sphincter function. If the urethra length can be maintained to at least $3-4 \mathrm{~cm}$ by reconstructing the urethra from the anterior bladder, this may offer some benefit. ${ }^{6}$ Combining this technique with a sling of pubococcygeal muscle seems hopeful.Although we found that the number of complex repairs previously performed by surgeons was not a significant predictor of repair outcomes, clearly, specialized skills are required for fistula surgery. All surgeries were conducted by trained fistula surgeons or by a trainee under the direct guidance of an experienced fistula surgeon. Surgeons who reported having conducted more than 200 complex repairs were repairing fistulas with a worse prognosis.

While the analyses controlled for a variety of fistula characteristics we cannot exclude the possibility of residual confounding by prognosis. Some limitations should be kept in mind. Although we accounted for a wide variety of patient and fistula characteristics and several contextual factors, the study was observational and the results may be subject to confounding by factors that were not measured or controlled for. Sociodemographic, obstetric history and causative delivery data were subject to the inaccuracies and reporting biases inherent in self-reported data. Laboratory measurements for some comorbidities and standard definitions for some fistula characteristics were not available, which may have led to underreporting or bias in our results. We believe that errors in exposure measurement were non-differential, and that any bias would be toward the null.Loss to follow-up varied across sites and our results may have been biased if women who completed the study differed from those who were lost; while we cannot rule out this possibility, 
loss to follow-up was remarkably low. Given the exploratory nature of the study, we may not have had sufficient power to detect small significant differences in some analyses. This study is one of the significant collections of data assessing fistula repair outcomes, and one of few studies that followed women after discharge to determine predictors of longer-term repair outcomes. The high follow-up rate suggests that it might be possible to assess even longer-term outcomes in future studies with fistula clients.Our results may help surgeons to make decisions about the skill level needed to repair individual patients as well as to communicate adequately to fistula patients about the possibility of a failed repair or remaining residual incontinence given the characteristic of their fistula. They also provide further evidence to support inclusion of certain fistula characteristics, particularly vaginal scarring and urethral involvement, in prognostic classification.

\section{References:}

1. AbouZahr C. Prolonged and obstructed labor. In: Murray CLA, editor. The health dimensions of sex and reproduction: the global burden of sexually transmitted diseases and HIV, maternal conditions, perinatal disorders and congenital anomalies. Boston, MA: Harvard University Press; 1998;242-266.

2. Waaldijk K, Armiya'u YD. The obstetric fistula: A major public health problem still unsolved. International Urogynecology Journal. 1993;4:126-128.

3. Wall LL. Obstetric vesicovaginal fistula as an international public-health problem. Lancet. 2006;368:1201-1209.

4. Frajzyngier V, Ruminjo J, Barone MA. Factors influencing urinary fistula repair outcomes in developing countries: a systematic review. Am J Obstet Gynecol. 2012;207(4):248-258.
5. Kayondo M, Wasswa S, Kaban.kyenga J, et al. Predictors and outcome of surgical repair of obstetric fistula at a regional referral hospital, Mbarara, western Uganda. BMC Urol. 2011;11:23.

6. Munoz O, Bowling CB, Gerten KA, et al. Factors influencing post-operative short-term outcomes of vesicovaginal fistula repairs in a community hospital in Liberia. 2011;4:259-265.

7. Sjoveian S, Vangen S, Mukwege D, Onsrud M. Surgical outcome of obstetric fistula: a retrospective analysis of 595 patients. Acta Obstet Gynecol Scand. 2011;90:753-60.

8. Goh JT, Browning A, Berhan B, Chang A. Predicting the risk of failure of closure of obstetric fistula and residual urinary incontinence using a classification system. Int Urogynecol J Pelvic Floor Dysfunct. 2008;19:1659-1662.

9. Raassen TJ, Verdaasdonk EG, Vierhout ME. Prospective results after first-time surgery for obstetric fistulas in East African women. Int Urogynecol J Pelvic Floor Dysfunct. 2008;19:73-79.

10. Kirschner C, Yost K, Du H, Karshima J, Arrowsmith S, Wall L. Obstetric fistula: the ECWA Evangel VVF Center surgical experience from Jos, Nigeria. International Urogynecology Journal. 2010;21:1525-1533.

11. Lewis A, Kaufman MR, Wolter CE, et al. Genitourinary fistula experience in Sierra Leone: review of 505 cases. J Urol. 2009;181:1725-1731.

12. Browning A. Risk factors for developing residual urinary incontinence after obstetric fistula repair. BJOG. 2006;113:482-485.

13. Nardos R, Browning A, Chen CC. Risk factors that predict failure after vaginal repair of obstetric vesicovaginal fistulae. Am J Obstet Gynecol. 2009:200(5):578. e1-4. 